\title{
On the strain rate limits in dynamic testing of concrete-like heterogeneous materials
}

\author{
Y. Lu and Z.H. Song \\ Institute for Infrastructure and Environment, School of Engineering, University of Edinburgh, \\ William Ranking Building, The King's Buildings, Edinburgh EH9 3JL, UK
}

\begin{abstract}
The bulk dynamic behaviour of concrete in generally known to be strain rate dependent. However, explanation of the underlying mechanisms has been a subject of debate in the research community. Among other factors, the extent of validity of existing experimental data for concrete-like materials in the high strain rate regime is believed to have further complicated the situation. This paper is concerned about the strain rate limits for dynamic testing of concrete specimens in association with the size requirement for representation of the composite material. The strain rate limits as may be established by elastic theories are discussed firstly. Representative simulation results from numerical experiments using a mesoscale model are then described, and non-uniform distributions of stress and damage under excessively high strain rates are highlighted, and this leads to discussion on the correlation between the externally measured data and the real responses within the sample material. Both dynamic compression and dynamic splitting tension are considered.
\end{abstract}

\section{Introduction}

The apparent strength of concrete-like materials is generally known to increase as the strain rate increases, although explanations of the underlying mechanisms may differ. In physical experiment, the determination of the apparent dynamic strength of the material is normally carried out through dynamic tests using a Split Hopkinson Pressure Bar or similar apparatus on standard sample specimens of certain dimensions. A key requirement for a valid SHPB type of tests is that relative stress uniformity is achieved before the specimen reaches a failure state [1,2], such that the externally observed (or inferred) strength and deformation at failure may be considered as representative of the bulk material behaviour. For testing into high strain rate regime (e.g. $100 \mathrm{~s}^{-1}$ and higher), this would necessitate the use of small size specimens within a few centimetre range. While small size specimens can be easily catered to for metallic materials, it poses a dilemma when comes to non-metallic, non-homogeneous materials such as concrete and rocks, which would generally require samples of a sufficient size so as to contain an enough number of aggregates for an appropriate representation of the composite behaviour.

While the requirement for the stress uniformity in SHPB tests is understood and generally recognised in the dynamic research community, the need to cater for the heterogeneity often dictates the use of large specimen sizes (e.g. [3-6]), without always observing a strain rate limit that is associated with guaranteeing stress uniformity. Among other factors, this is believed to have contributed, to a varying extent, to the large scatter in the observed dynamic strength increase factor (DIF) for compressive as well as tensile strengths.

In this paper, we shall take a close look at the response and failure processes of concrete specimens in association with the strain rate limit using numerical experiments for both compression and tension (spit) conditions. We shall highlight that as the strain rate limit is exceeded, the external measurements would no longer be able to represent the internal material behaviour in a way as presumed in an ideal condition. In the event of non-compliance with the strain rate limit, appropriate evaluation over the sample structural effect could help extract useful information while avoiding incorrect or misleading interpretation from the recorded data.

\section{Strain rate limit for compression}

\subsection{Strain rate limit}

Two basic assumptions should be satisfied in order for a SHPB test to be strictly valid, a) one dimensional elastic wave propagation within the pressure bars and the specimen, and b) uniaxial and uniform stress state within the specimen. To meet the second requirement, several reflections of the stress wave are needed; thus the minimum time required for the stress to reach equilibrium may be written as:

$$
t_{m}=n \frac{l_{s}}{c_{0}}
$$

where $l_{s}$ is the length of specimen, $c_{0}$ is the sound wave speed in the specimen, $n$ is the number of wave reverberations, and is recommend to be 3 4 based on experimental observations $[1,7]$. Accordingly, an upper limit of strain rate can be established as:

$$
\dot{\varepsilon}_{m}=\frac{\varepsilon_{c}}{\alpha t}=\frac{\varepsilon_{c} c_{0}}{\alpha n l_{s}}
$$

where $\varepsilon_{c}$ denotes the strain at the maximum strength, $\alpha$ is an integration (strain rate rise) coefficient. $\alpha$ would normally lie in a range of 0.5 to 1.0 (1.0 for an idealised situation with zero rise time, while 0.5 corresponding to a linear rise until failure, but in that case more wave reverberations may be required to reach stress uniformity at a desired level). 


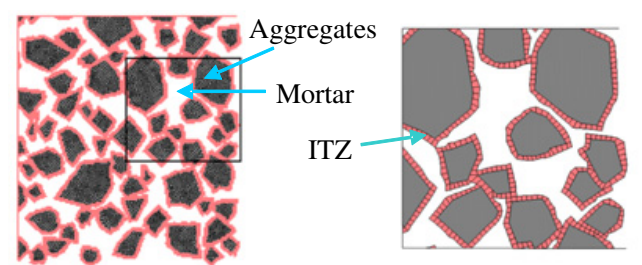

(a) Partial meso structure

(b) Close-up ITZ mesh

Fig. 1. Configuration of the mesoscale model.

Take 30-MPa concrete for example, consider a peak strain 0.002 , mass density $2300 \mathrm{Kg} / \mathrm{m}^{3}$, and Young's modulus $20 \mathrm{GPa}$, a standard $50-\mathrm{mm}$ long specimen would require a uniformity build-up time $\left(\mathrm{t}_{\mathrm{m}}\right)$ of about $0.05 \mathrm{~ms}$, and a corresponding strain rate limit of order of $50 \mathrm{~s}^{-1}$.

Numerous data points towards the high strain-rate end in the existing dataset in the literature (see e.g. [2]) appear to fall beyond the upper strain-rate limits for the respective specimens. For example in reference [3] concrete specimens of length $51 \mathrm{~mm}$ were used; theoretically this would restrict the valid strain rate range to be below $40 \sim 50 \mathrm{~s}^{-1}$; however, the actual tests extended to a strain rate as high as $1000 \mathrm{~s}^{-1}$. Investigating issues associated with exceeding the strain rate limit is of apparent practical relevance.

\subsection{Pulse (strain rate) rise time}

Another closely related factor is the rise time of the load pulse, which may be translated into the boundary velocity or a nominal strain rate rise. Increasing the rise time is generally deemed to improve the condition for stress uniformity, whereas decreasing the rise time could subject the sample to unwanted local damage. However, because the failure strain of a material sample is definite (for a certain strain rate), the rise time cannot be artificially prolonged beyond a certain limit; otherwise the material would reach failure before the target strain rate could be attained. Further discussion on the rise time will be given later in discussing the split tension tests.

\section{Numerical simulation of dynamic compression with a mesoscale model}

\subsection{Overview of the mesoscale model}

When strain rate is high, drastic variation (i.e. large gradient) of stress and strain occur as high intensity stress wave reverberate within a specimen. To realistically represent response of concrete under high stress/strain gradient, an explicit inclusion of the mesoscale heterogeneity becomes crucial. Herein a mesoscale model incorporating random polygon-shaped aggregates and suited for dynamic analysis [8] is employed to examine the response of concrete samples in association with the strain rate limits. Fig. 1 shows a sample 2D mesoscale structure and the finite element mesh. Different material properties are used for the three individual phases. The mortar matrix and the ITZ, which is represented by a thin layer of solid elements, are

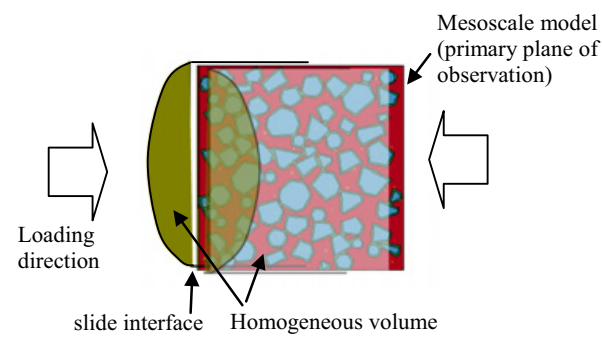

Fig. 2. Pseudo 3D mesoscale model for a cylindrical sample.

modelled using a damage-plasticity model. The aggregates are modelled as nonlinear material with high strength (150MPa herein) that represents typical crushed granite.

For the compression analysis, it is important that the potential lateral inertial confining effect be appropriately represented in the model. In a $2 \mathrm{D}$ homogeneous model of a cylindrical specimen, this can be achieved by employing an axis-symmetrical setting. However, when the meso structure is involved, a 2D axis-symmetric model would effectively render aggregates as "aggregate rings" which would impose false confinement as the sample tends to expand. To resolve this problem while maintaining a realistic 3D inertia environment, a sandwich model configuration is put forward to cater for the mesoscale analysis using a 2D mesoscale representation for a "3D" compression analysis [8]. Fig. 2 illustrates the model configuration.

\subsection{Compression simulation}

A cylindrical specimen of $50 \times 50 \mathrm{~mm}$ is considered for the analysis. The maximum aggregate has a nominal size of $8 \mathrm{~mm}$. Examination of the responses is focused within the mesoscale plane of the pseudo 3D model. To facilitate the evaluation of the stress-strain distribution while the stress wave propagates and reflects between the two end faces, the mesoscale plane is fictitiously divided into five equal strips (strips 1-5) along the loading (axial) direction. To simplify the analysis, the imposed loading is simulated via prescribed time varying boundary condition at the loading (left) face, with an idealised velocity history consisting of a gradual rise phase followed by a constant velocity.

To facilitate the discussion on the dynamic structural effect, no strain rate enhancement (DIF) is enforced at the material constitutive model level. Comparative results for two nominal strain rates, namely $50 \mathrm{~s}^{-1}$ which represents a valid strain rate for the size of sample, and $200 \mathrm{~s}^{-1}$ which represents an "excessive" strain rate beyond the theoretical limit are examined here.

Fig. 3 shows the development of axial stress in the five consecutive strips for the above two strain rates, respectively, along with the corresponding damage patterns. Under the strain rate of $50 \mathrm{~s}^{-1}$, all strips appear to reach the maximum strength more or less at the same time. This suggests that a relatively uniform stress state can indeed be achieved when the applied strain rate is generally within the theoretical limit. In fact from the stress histories it can be observed that two complete reverberations have occurred before the maximum strength is reached. The damage patterns also demonstrate a relatively uniform 

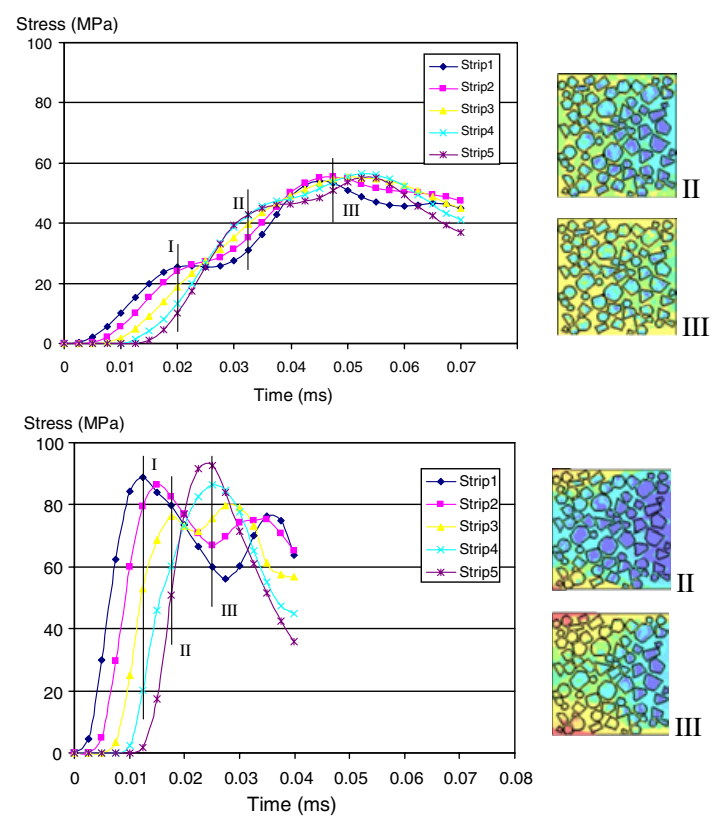

Fig. 3. Stress time histories in individual strips; upper $=50 \mathrm{~s}^{-1}$, lower $=200 \mathrm{~s}^{-1}$.

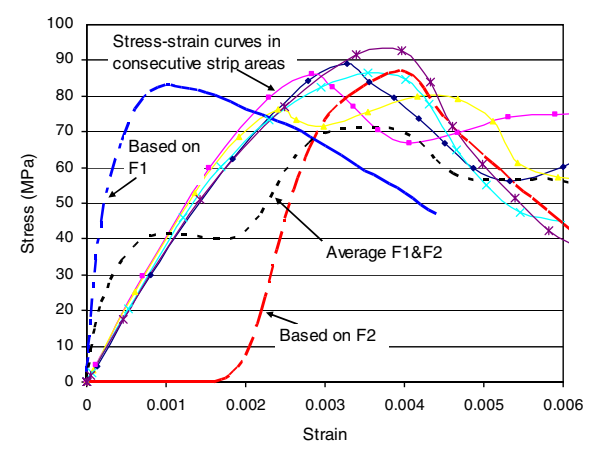

Fig. 4. Stress-strain curves inferred with different approaches for the strain rate case of $200 \mathrm{~s}^{-1}$.

damage. For the "excessive" strain rate case of $200 \mathrm{~s}^{-1}$, however, failure apparently initiates as soon as the first stress wave is encountered at a particular location, as can be seen from the stress histories and the damage contours. No overall stress uniformity or static equilibrium is developed.

Examining the axial stress, in can be found that the dynamic strength achieved in individual strips increases with the increase of the strain rate, despite that no rate sensitivity is enabled at the material level. The dynamic strength increase works out to match closely the empirical DIF such as the CEB model. Similar to the observations made in previous studies (e.g. [5,9]), such an increase is found to be attributable to the increase in the confining stress, due primarily to the lateral inertia, combined with the pressure sensitive feature of the material strength. That is to say, the dynamic structural effect plays a dominant role in the apparent strength increase of the sample in compression.

Fig. 4 shows the strip-wise stress-strain curves for the $200 \mathrm{~s}^{-1}$ case. The stress-strain curves are observed to be consistent among different strips, even for this excessive strain rate case. Comparison with the curves for the other strain rates (not shown), the Young's modulus is almost independent of the strain rate. As a result, the strain at the peak strength increases almost proportionately as the peak strength increases with the strain rate.

To correlate with what one can obtain from typical SHPB measurements, the global (sample-wide) nominal stress and strain responses in the mesoscale numerical model are deduced using quantities at the ends faces (as if being inferred from incident and transmitter bars) in accordance with the classical 1-wave, 2-wave, or 3wave method [7]. The results of the so obtained global (engineering) stress-strain curves are also compared with the strip-wise results in Fig. 4 for the $200 \mathrm{~s}^{-1}$ case. While for the $50 \mathrm{~s}^{-1}$ case (not shown) the global stress-strain relationship curves using the three different wave methods exhibit consistent outcome in terms of the peak stressstrain and resemble reasonably the strip-wise curves, for the case with $200 \mathrm{~s}^{-1}$, the engineering stress-strain curves deviate considerably from the "true" stress-strain results at the local material level. More specifically, the inferred strain response appears to be uncorrelated with the actual strain inside the sample domain. Consequently, the Young's modulus is considerably overestimated, while the peak strain is significantly underestimated.

It is worth pointing out, however, that the processed dynamic strength using the 1-wave or 2-wave approach reasonably captures the peak dynamic strength of the sample material.

\section{Strain rate limit in split tension}

\subsection{General considerations}

Splitting tests in a Brazilian disk configuration are commonly used in determining the tensile strength of concretelike materials under quasi-static loading:

$$
\sigma_{t}=\frac{2 P}{\pi L D}
$$

where $P$ is the compression force applied on the loading strip, D is the diameter, and L is the thickness (length) of the disk.

When this technique is applied in high strain rate tests using a SHPB apparatus, the basic assumption is that an equilibrium condition similar to that under static loading can be attained before the test specimen reaches the tension failure. The applied forces at the loading (incident) and supporting (transmitter) ends can be determined from the strains in the incident and transmitter bars, and subsequently the splitting tensile stress can be calculated by Eq. (3). The achieved tensile strain can be evaluated from the calculated tensile stress time history:

$$
\dot{\varepsilon}_{t}=\frac{\Delta \sigma_{t}}{E_{c}(\Delta t)}
$$

where $\Delta \sigma_{t}$ denotes the increment of the tensile stress over a time duration $\Delta t, E_{c}$ is the Young's modulus of concrete. 
Similar to the situation with dynamic compression, establishment of an equilibrium condition requires a minimum lead time for the stress to build up and the transient effect to decay, which implies a strain rate limit. In fact the rate limit requirement in a splitting tension test can be more stringent than in compression as the inference of the tensile stress is indirectly made through the splitting compression force. If the required equilibrium condition is not achieved the calculated tensile stress could simply be invalid and misleading. Rodriguez et al. [10] carried out a simple but quite illustrative elastic FE analysis on a specimen representing a ceramic material, with Young's modulus $200 \mathrm{GPa}$, Poisson's ratio 0.33 and density $2690 \mathrm{~kg} / \mathrm{m}^{3}$. The specimen had a dimension of $10 \mathrm{~mm}$ in height and 20 $\mathrm{mm}$ in diameter. The dynamic load was applied through imposing a trapezoidal velocity history to one end of the specimen with a maximum value of $13 \mathrm{~m} / \mathrm{s}$ and a rise time of $20 \mu$ s, as obtained from their Hopkinson bar tests. It was found that $8 \mu$ s was minimum to complete the process of a few stress wave reverberations to reach a symmetrical distribution and an equilibrium state. After that time, the tensile stress in the splitting region was well represented by Eq. (4).

A similar deduction may be made on a typical concrete specimen of $50 \mathrm{~mm}$ in diameter to yield a build-up time of around $50 \mu \mathrm{s}$ (which is consistent with the estimation of $\mathrm{t}_{\mathrm{m}}$ for compression) for the development of a uniform stress distribution. From here an estimate about the tensile strain rate limit may be made that would permit $50 \mu$ s of loading time before reaching the tensile failure strain. Assuming a gradual increase of the loading (strain) rate until the dynamic tensile strength is attained, it leads to:

$$
\dot{\varepsilon}_{t, \max }=\frac{2 \varepsilon_{d t}}{\tau}
$$

where $\varepsilon_{d t}$ is the quasi-static tensile failure strain, and $\tau$ denotes the duration of loading before failure. For example, let us assume a dynamic tensile failure strain of $250 \mu \varepsilon$, with $\tau=50 \mu \mathrm{s}$, a limit strain rate would be on an order of $10 \mathrm{~s}^{-1}$. Even for smaller cylinders, for example with a diameter of $10 \mathrm{~mm}$ for a mortar sample, the maximum allowable strain rate would still be just about $50 \mathrm{~s}^{-1}$.

\subsection{Numerical simulation of splitting tension}

A few simulation examples are given in this section to illustrate the feasibility of achieving a wide range of strain rates while attempting to maintain a desirable stress/ equilibrium condition by involving a sufficiently long rise phase. Loading is applied via a velocity boundary on the splitting strip at the loading side, see Fig. 5. The velocity scheme has a gradual rise stage followed by a constant phase. Three target strain rates are investigated, namely 1 $\mathrm{s}^{-1}$ (within the strain rate limit), $10 \mathrm{~s}^{-1}$ (around the strain rate limit), and $50 \mathrm{~s}^{-1}$, corresponding respectively to a velocity of $0.25,2.5$ and $12.5 \mathrm{~m} / \mathrm{s}$.

Figs. 6-7 show the time history of boundary force $\mathrm{P} 1$, distribution of the tensile (y-direction) stress along the loading diameter prior to initiation of failure, and the damage (fracture) contour for the $10 \mathrm{~s}^{-1}$ and " 50 " $\mathrm{s}^{-1}$ strain rate cases.
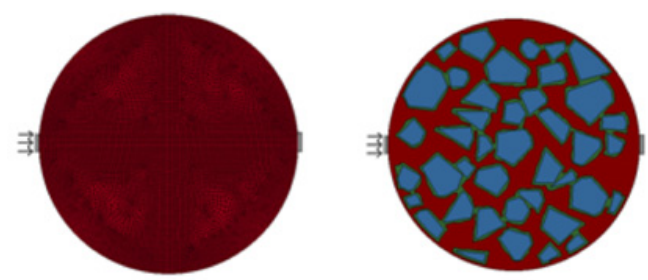

Fig. 5. Model configuration for dynamic splitting tension simulation.

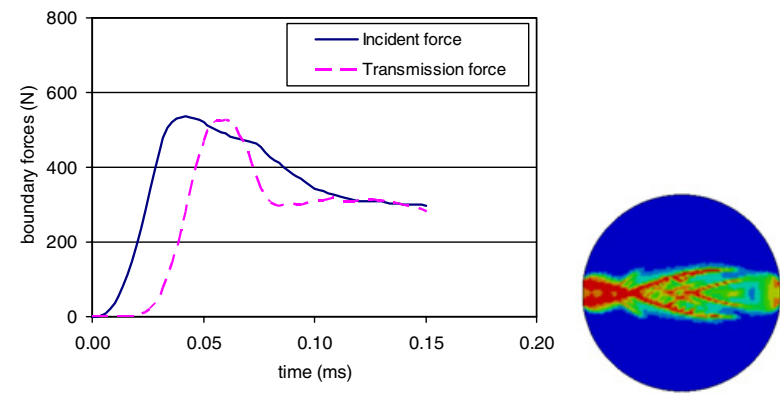

(a) Force P1 history

(b) Damage contour

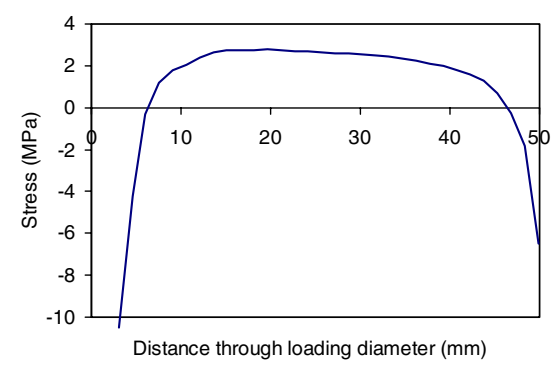

(c) Tensie (y-) stress distribution along spitting line

Fig. 6. Split tension response to nominal strain rate $10 \mathrm{~s}^{-1}$.

It can be observed that for the $1 \mathrm{~s}^{-1}$ strain rate case (not shown) a uniform tensile stress is developed well before the initiation of failure, which occurs at about $0.2 \mathrm{~ms}$. A clear splitting zone is developed as can be expected from a standard splitting test. A generally similar situation is observed for the strain rate $10 \mathrm{~s}^{-1}$ case (Fig. 6). However, under this rate the tensile stress distribution is just about uniform when failure starts to occur at about $0.04 \mathrm{~ms}$. The damage contour also exhibits a somewhat non-symmetric pattern with heavier damage towards the loading side. As the maximum load is reached close to the onset of the constant velocity $(0.04$ against $0.05 \mathrm{~ms})$, the target nominal strain rate of $10 \mathrm{~s}^{-1}$ is almost achieved.

The situation with the $50 \mathrm{~s}^{-1}$ rate case is very different. First of all, the maximum load occurs at about $0.02 \mathrm{~ms}$, which is well within the rise phase and corresponds only to a velocity of less than half of the target value. This indicates that the sample fails at a (nominal) strain rate much lower than the target strain rate of $50 \mathrm{~s}^{-1}$. Secondly the tensile stress distribution is highly non-uniform, and apparently no equilibrium state is reached. It may therefore be concluded that the situation is simply not a valid splitting case. This result further confirms the earlier argumentation about the required time (of about $0.05 \mathrm{~ms}$ 

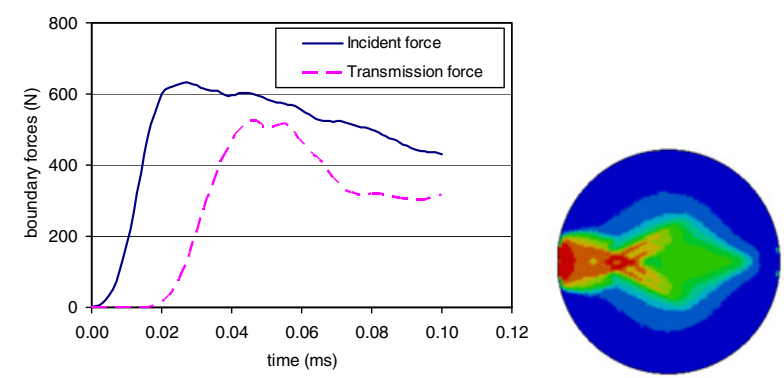

(a) Force P1 history

(b) Damage contour

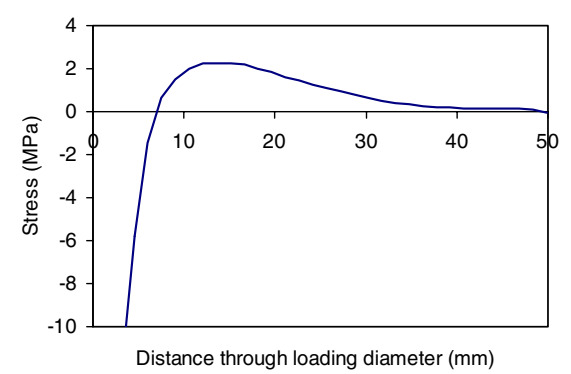

(c) Tensie (y-) stress distribution along spitting line

Fig. 7. Split tension response to nominal strain rate " $50 " \mathrm{~s}^{-1}$.

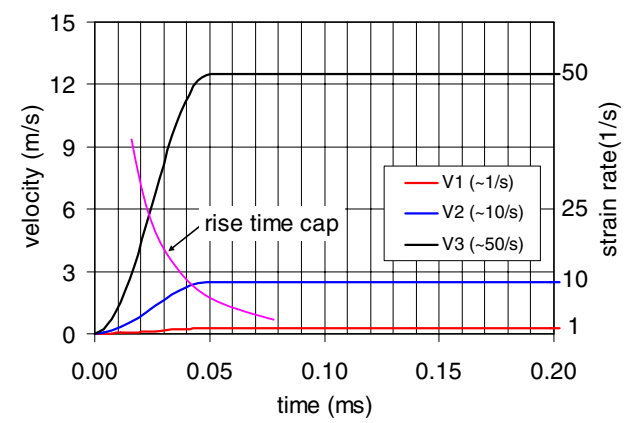

Fig. 8. A schematic illustration of strain rate - rise time cap.

herein) to establish a valid splitting equilibrium state. If failure initiates in less than this amount of time, albeit the provision of a sufficiently long rise time in the load pulse, complications arise.

Fig. 8 shows schematically a "cap" that limits the effective rise time as a function of a target strain rate. The exact position of the cap curve may vary to a certain extent considering variation of the failure strain for different strain rates; however the general trend should hold such that it is not possible to attain a high strain rate and at the same time ensure stress uniformity just by adopting a prolonged rise time. The strain rate limit as set out by the stress build-up requirement, in this case about $10 \mathrm{~s}^{-1}$, will tend to prevail.

In connection with physical spitting tests, the rise time is usually set in a range of $0.04-0.1 \mathrm{~ms}$ (see e.g. [11-13]. Based on the discussion herein, the actually achievable strain rate would have been limited to no more than about $10 \mathrm{~s}^{-1}$ with such a range of rise time.

The opposite situation to what is discussed above would be an attempt to promote the attainment of a high strain rate before a failure strain by adopting a small rise

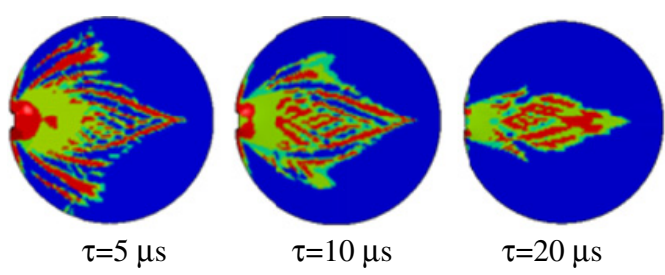

Fig. 9. Damage patterns for a target strain rate of $50 \mathrm{~s}^{-1}$ but with different rise times.

time. This is obviously not a valid approach in view of the required time (number of wave reverberations) to reach a stress uniformity/equivalent state; and moreover, in a splitting test this would also increase the likelihood of local failure near the loading strip, thus further distort a split-tension condition. This can be clearly observed from Fig. 9, in which the same target strain rate of $50 \mathrm{~s}^{-1}$ is attempted with a shortened rise time. It can be observed that the specimen is subject to severe local impact damage when the rise-time is less than $0.02 \mathrm{~ms}$.

The simulation results also allow for a general observation of the dynamic structural effect in the split tension tests. As mentioned earlier, the material is modelled as strain-rate insensitive. From the force results shown in Figs. 6-7, there appear to be no significant increase in the achieved splitting forces for the $50 \mathrm{~s}^{-1}$ case as compared to the $10 \mathrm{~s}^{-1}$ case. This is contrary to the observation made in compression simulations, where persistent increase is found in the dynamic force as the strain rate increases. It can be deduced from here that the significant increase in the P1 force as observed in physical spitting tests would not have been possible if the tensile strength at the composite material level did not increase with the strain rate. Similar conclusions have been made in some previous studies [11,12]. An extended discussion on this topic is beyond the scope of the present paper.

It is also worth noting that if a steep rise phase is to be employed, some significant increase in the splitting force may be obtained but that is more of a dynamic compression effect than a valid dynamic structural effect on the splitting tensile strength.

The simulation of splitting tension has also been carried out using the mesoscale model. The general fracture patterns from the mesoscale analysis agree with those from the homogeneous models; however the detailed distributions are apparently affected by the presence of the aggregates. Comparison of the failure splitting forces with the results from the homogeneous models indicate that the presence of the stronger aggregates tend to introduce an appreciable amount of increase in the dynamic splitting tensile strength. For the range of strain rates investigated this increase ranges on an order of $20 \%$.

\section{Conclusions}

Stress uniformity and overall equilibrium constitute the general requirements for a valid dynamic tests using SHPB type of apparatus. However, it is not always possible for such requirements to be strictly adhered to in dynamic 
tests of concrete-like materials, especially in the high strain rate regime. Understanding of the potential deviations of response from those under the ideal conditions can be vital for appropriate extraction and interpretation of the test results.

In dynamic compression, numerical results indicate that in the event of exceeding the strain rate limit, the dynamic strength measured from external forces will tend to resemble the actual strength occurred inside the sample specimen; however the nominal strain will tend to be much lower than the actual strain, resulting in overestimation of the Young's modulus. Further complications can arise due to strain rate variation within the sample specimen when the strain rate limit is not observed. In dynamic split tension, exceeding the strain rate limit would effectively invalid the whole split tension condition.

Results from the mesoscale numerical analysis also demonstrate that the compressive DIF is largely attributable to the dynamic structural effect, particularly the lateral inertial confinement, with an appreciable contribution from the material heterogeneity. On the contrary, the dynamic tensile strength tends to be primarily attributable to the "material" dynamic strength, including the contribution of the heterogeneity.

\section{References}

1. E.D.H. Davies, S.C. Hunter, The dynamic compression testing of solids by the method of the split Hopkinson pressure bar, J. Mech. Phys. Solids. 11: 155-179 (1963).

2. P.H. Bischoff, S.H. Perry, Compression behaviour of concrete at high strain-rates, Mater Struct. 24: 425450 (1991).

3. C.A. Ross, J.W. Tedesco, S.T. Kuennen, Effects of strain rate on concrete strength, ACI Mate J. 92: 75-81 (1990)
4. D.L. Grote, S.W. Park, M. Zhou, Dynamic behavior of concrete at high strain rates and pressures: I. Experimental characterization, Int J Impact Eng. 25: 869-86 (2001).

5. Q.M. Li, H. Meng, About the Dynamic strength enhancement of concrete like materials in a split Hopkinson pressure bar test, Int J Solids Struct., 40: 343-360 (2003)

6. X.Q. Zhou, H. Hao, Modelling of compressive behaviour of concrete-like materials at high strain rate, Int. J Solids Struct. 45: 4648-61 (2008).

7. P.S. Follansbee, The Hopkinson pressure bar, in: Kuhn, H, Medlin, D. (Eds), ASM Handbook-Vol. 8 Mechanical Testing and Evaluation. ASM International; 198-203 (1985).

8. Y. Lu, Z.H. Song, Z.G. Tu, Analysis of dynamic response of concrete using a mesoscale model incorporating 3D effects, Int J Prot Struct., 1(2): 197-217 (2010).

9. F.V. Donze, S.-A. Magnier, L. Daudeville, C. Mariotti, C. Davenne, Numerical study of compressive behavior of concrete at high strain rates, J Eng Mech-ASCE. 125: 1154-63 (1999).

10. J. Rodriguez, C. Navarro, V. Sanchez-Galvez, Splitting tests: an alternative to determine the dynamic tensile strength of ceramic materials. Journal de Physique IV, 4(C8): 101-106 (1994).

11. G. Ruiz, M. Ortiz, A. Pandol, Three-dimensional finite-element simulation of the dynamic Brazilian tests on concrete cylinders. Int. J. Numer. Meth. Engng, 48, 963-994 (2000).

12. M.L. Hughes, J.W. Tedesco, C.A. Ross, Numerical analysis of high strain rate slitting-tensile tests. Computers \& Structures, 47(4/5): 653-671 (1993).

13. J.W. Tedesco, C.A. Ross, S.T. Kuennen, Experimental and numerical-analysis of high-strain rate splitting tensile tests. ACI Materials Journal, 90(2), 162-169 (1993). 